\title{
Drechslerella stenobrocha genome illustrates the mechanism of constricting rings and the origin of nematode predation in fungi
}

Keke Liu ${ }^{1,2 \dagger}$, Weiwei Zhang ${ }^{1,2 \dagger}$, Yiling Lai ${ }^{1,2}$, Meichun Xiang ${ }^{1}$, Xiuna Wang ${ }^{1}$, Xinyu Zhang ${ }^{1}$ and Xingzhong Liu ${ }^{1 *}$

\begin{abstract}
Background: Nematode-trapping fungi are a unique group of organisms that can capture nematodes using sophisticated trapping structures. The genome of Drechslerella stenobrocha, a constricting-ring-forming fungus, has been sequenced and reported, and provided new insights into the evolutionary origins of nematode predation in fungi, the trapping mechanisms, and the dual lifestyles of saprophagy and predation.

Results: The genome of the fungus Drechslerella stenobrocha, which mechanically traps nematodes using a constricting ring, was sequenced. The genome was $29.02 \mathrm{Mb}$ in size and was found rare instances of transposons and repeat induced point mutations, than that of Arthrobotrys oligospora. The functional proteins involved in nematode-infection, such as chitinases, subtilisins, and adhesive proteins, underwent a significant expansion in the A. oligospora genome, while there were fewer lectin genes that mediate fungus-nematode recognition in the D. stenobrocha genome. The carbohydrate-degrading enzyme catalogs in both species were similar to those of efficient cellulolytic fungi, suggesting a saprophytic origin of nematode-trapping fungi. In D. stenobrocha, the down-regulation of saprophytic enzyme genes and the up-regulation of infection-related genes during the capture of nematodes indicated a transition between dual life strategies of saprophagy and predation. The transcriptional profiles also indicated that trap formation was related to the protein kinase $C$ (PKC) signal pathway and regulated by $\mathrm{Zn}(2)-\mathrm{C} 6$ type transcription factors.
\end{abstract}

Conclusions: The genome of $D$. stenobrocha provides support for the hypothesis that nematode trapping fungi evolved from saprophytic fungi in a high carbon and low nitrogen environment. It reveals the transition between saprophagy and predation of these fungi and also proves new insights into the mechanisms of mechanical trapping.

Keywords: Nematode-trapping fungi, Comparative genomic analysis, Origin of nematode predation, Transcriptomes, Trapping mechanism

\section{Background}

Predation is one fungal life strategy to manipulate freeliving nematode dynamics in nature and capture nitrogen [1-3]. Fungi have evolved sophisticated trapping structures, including constricting rings traps $[1,4]$ and five types of adhesive traps (sessile adhesive knobs, stalked adhesive knobs, adhesive nets, adhesive columns, and non-constricting rings) [5,6], with which they capture nematodes for nutritional purpose $[7,8]$. Based on molecular clock and fossil

\footnotetext{
*Correspondence: liuxz@im.ac.cn

${ }^{\dagger}$ Equal contributors

'State Key Laboratory of Mycology, Institute of Microbiology, Chinese Academy of Sciences, No 3 1st Beichen West Rd., Chaoyang District, Beijing 100101, China Full list of author information is available at the end of the article
}

evidence, nematode predatory ascomycetes were estimated to have originated as a result of mass extinctions in the Permian and Triassic $[9,10]$. The hypothesis holds that dead creatures caused by mass extinctions were rich in carbon but poor in nitrogen, so direct capture of nitrogen rich living animals would give predatory fungi a competitive advantage over strictly saprophytic fungi [11]. Constricting rings is considered to be the ancestral strategy after which all of the adhesive traps evolved [6].

Most nematode-trapping fungi can live both saprophytically on organic matter and as predators by capturing tiny animals [12]. Abundant nematode-trapping fungi have been detected in niches that that are rich in decayed organic matter, such as decayed leaves and wood [13]. 
Nematode-trapping fungi that form constricting rings have high density in the soil with rich organic matters whereas the fungi that form adhesive nets mostly diversity in the relatively poorer soil [14]. They are also influenced by the population density of nematodes, which are key nutritional resources for these fungal populations [15].

Traps are usually produced from hyphae in the presence of nematodes [4] or are induced by peptides, or nematode extracts from the nematodes [16]. Drechslerella stenobrocha (Ascomycota: Orbiliaceae) is a nematode-trapping species that forms constricting rings, the most sophisticated trapping structure, that consist of three ring cells capturing nematodes actively (Figure 1A, B) [17]. When a nematode enters the ring and contacts the inner surfaces of the ring cells, G protein-coupled receptors activate a down-stream signal pathway that includes cyclic adenosine monophosphate (cAMP), inositol-1, 4, 5-triphosphate (IP3), and $\mathrm{Ca}^{2+}[18]$. Subsequently, the ring cells rapidly (within $0.1 \mathrm{~s}$ ) triple their volume and firmly lasso the nematode (Figure 1C, D) [19].

Arthrobotrys oligospora (Ascomycota: Orbiliaceae) is an adhesive network-forming nematode-trapping fungus and a model organism for understanding the interactions between fungi and nematodes [20]. It can also parasitize other fungi and colonize plant roots $[5,16,21]$. Its genome sequence, the first available for a nematode-trapping fungus, provided information about some of the proteins responsible for trap formation, including G-protein coupled receptors, adhesive proteins, cell division cycle (CDC37), peroxisome-related proteins, and proteins involved in energy supplementation [22]. Although other fungal genomes, such as those of entomopathogenic fungi, have identified enzymes like subtilisins and chitinases to be important virulence factors in host barrier degradation [23,24], the available fungal genome data are not sufficient to elucidate the origin of fungal predation to nematodes or the trapping mechanisms. Since D. stenobrocha uses a constricting-ring mechanical trapping mechanism, the comparison of its genome with that of $A$. oligospora should understand the origin of nematode predation and the mechanisms.

To test the hypothesis that trapping fungi originated in carbon-rich but nitrogen-poor niches as well as to understand the nematode predatory lifestyle and constricting-ring trapping mechanism, the genome of D. stenobrocha was analyzed and compared with that of the networkforming fungus $A$. oligospora as well as those of nontrapping fungi. The results provide a comprehensive understanding of the biology of nematode-trapping fungi and crucial data for further studies of their trapping mechanisms.

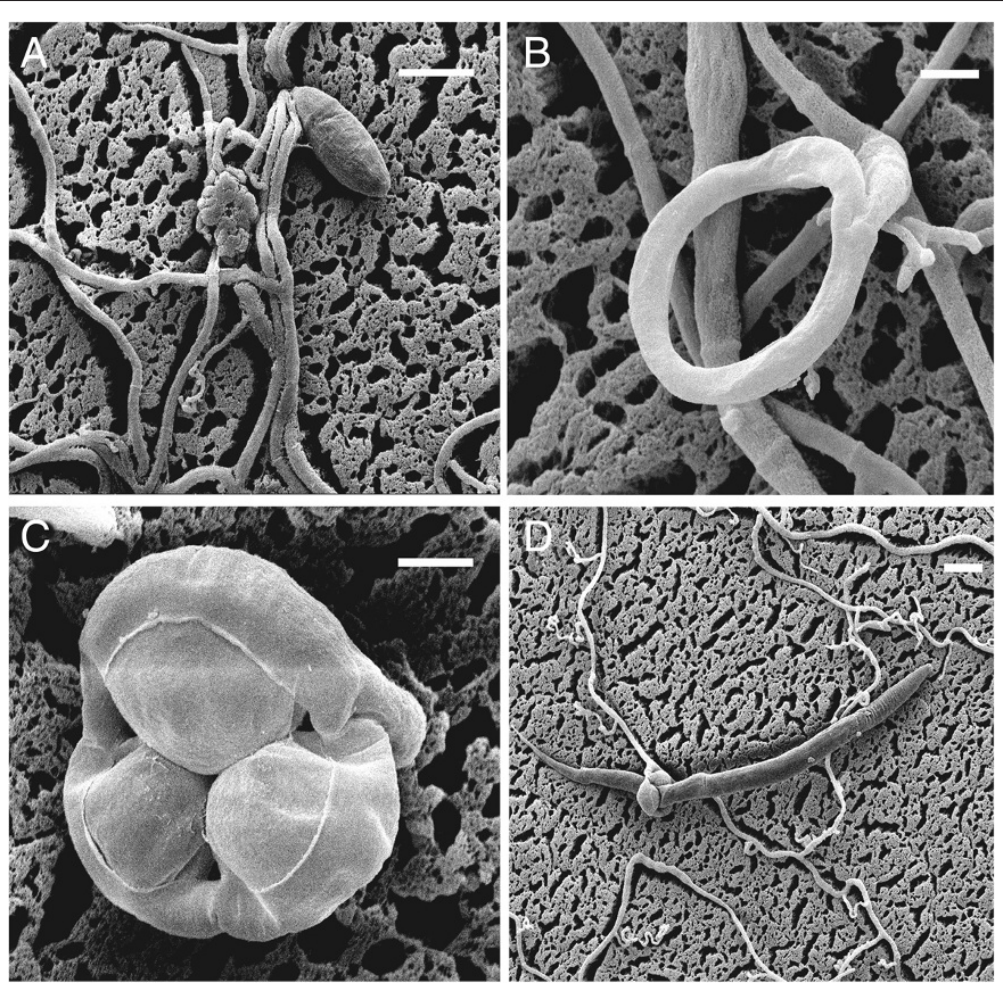

Figure 1 Characteristics of $D$. stenobrocha used in the sequenced nematode-trapping fungus, showing the amazing mechanical trapping process. The trapping process includes ring formation and ring constriction. A) The free living hyphae and spore; B) Ring without constriction; C) Ring induced to constrict; D) Nematode is capturing. Scale bar: $\mathbf{A}, \mathbf{D}=20 \mu \mathrm{m} ; \mathbf{B}, \mathbf{C}=5 \mu \mathrm{m}$. 


\section{Results}

The $D$. stenobrocha genome is smaller than that of the phylogenetically closely related $A$. oligospora

The 29.02-Mb genome of $D$. stenobrocha was sequenced with Solexa ( $\sim 80 \times$ coverage), with the NCBI accession of ASQI01000000. It yielded 142 scaffolds, 134 of which were 2-kb or larger, and had a repeat content of $0.97 \%$ (Table 1 ). In contrast, genome of $A$. oligospora was $40.07 \mathrm{Mb}$ in size, with 323 scaffolds, 252 of which were $2-\mathrm{Kb}$ or larger [22]. A total of 11,479 genes were predicted for A. oligospora, whereas only 7,781 genes, with few duplicates, were estimated in the genome of $D$. stenobrocha (Additional file 1: Figure S1, Additional file 2: Table 1). The two genomes shared 7,036 homologs with more than $80 \%$ predicted amino-acid similarity, covering more than $90 \%$ of the genes in the D. stenobrocha genome. However, this number dropped to 5,626-6,210 when comparing the $D$. stenobrocha or A. oligospora genomes with those of other selected species that include two entomopathogens (Metarhizium acridum and Metarhizium anisopliae), three phytopathogens (Magnaporthe oryzae, Verticillium albo-atrum and Fusarium graminearum), two saprobes (Aspergillus nidulans and Neurospora crassa), and one symbiotic fungus (Tuber melanosporum) (Figure 2A, Additional file 2: Table S1). More than 1,400 predicted genes matched strictly between $D$. stenobrocha and $A$. oligospora, indicating their close phylogenetic relationship. When D. stenobrocha was compared with other selected fungi, only 647 orphan sequences (without known homologs) were predicted in its genome, while the $A$. oligospora genome had 2,578 orphan sequences.

Phylogenetic analyses of $D$. stenobrocha, A. oligospora, and 10 other fungal species (Additional file 2: Table S1)

\begin{tabular}{ll}
$\begin{array}{l}\text { Table } 1 \text { Main features of the Drechslerella } \\
\text { stenobrocha genome }\end{array}$ \\
\hline Features & D. stenobrocha \\
\hline Assembled size (Mb) & 29.02 \\
Scaffolds (larger than 2 Kb) & 134 \\
Scaffolds (total) & 142 \\
Scaffold N50 (bp) & 434,454 \\
Coverage (fold) & $80 \times($ Solexa) \\
G + C content (\%) & 52.5 \\
Repeat rate (\%) & 0.92 \\
Coding rate (\%) & 41.55 \\
protein-coding genes & 7,781 \\
Gene density (genes per Mb) & 268.3 \\
GC exonic (\%) & 55.31 \\
GC intronic (\%) & 49.24 \\
Exons per gene & 3.57 \\
tRNA genes & 82 \\
\hline
\end{tabular}

also confirmed that the former two were much more closely related to one another than to the other sampled species. Drechslerella stenobrocha diverged 78-127 million years (MY) ago, earlier than the insect parasites Metarhizium anisopliae and Metarhizium acridum (26$34 \mathrm{MY}$ ), but later than the plant parasite Fusarium graminearum (200-260 MY) and the saprophyte Neurospora crassa (240-300 MY) (Figure 2B).

\section{Fewer repeat-induced point mutations and transposons}

in the $D$. stenobrocha genome

A lack of transposases ( 1 in D. stenobrocha versus 11 in A. oligospora) limited the occurrence of transposons (one transposon of $984 \mathrm{bp}$ ) in the D. stenobrocha genome, resulting in its smaller genome size. Like the fungi such as Verticillium dahliae and Verticillium albo-atrum [25], more than $95 \%$ of the repetitive sequences $(1.42 \%$ transposable elements) in the A. oligospora genome occurred in non-syntenic regions. The 256 transposable elements in the $A$. oligospora genome were classified into different repeat families. Class I retrotransposons comprised $50 \%$ of the repetitive fraction, unclassified transposable elements comprise almost all of the remainder.

Repeat-induced point (RIP) mutations, referred to as CpG to TpA mutations in duplicated sequences, are a defense mechanism to suppress the activity of transposases in fungal genomes. They were first described in the genome of Neurospora crassa [26,27]. The gene NCU02034 (http://www.ncbi.nlm.nih.gov/protein/AAM27408.1) in $N$. crassa, which is only known to be required for RIP, lack its homologous gene found in the $D$. stenobrocha genome [27]. In addition, RIPs occur only in repetitive regions larger than $500 \mathrm{~Kb}$ and no such repetitive sequences were found in D. stenobrocha. These indicate the lack of RIPs. However, significant evidence of RIPs was found in the A. oligospora genome, especially in the repeat-rich nonsyntenic regions, which might explain the arising of numerous orphan genes related to nematode-infection.

\section{Genes enriched in non-syntenic, repeat-rich regions are associated with fungal predation}

The repeat-rich but gene-poor regions were hypothesized to contain genes associated with host adaptations based on genome analyses of phytopathogenic fungi such as Phytophthora species and Leptosphaeria maculans [28,29]. Similar results were obtained for nematode-trapping fungi. The syntenic regions of $29 \mathrm{Mb}$ (Figure 3A) contained more than $80 \%$ of the predicted genes in A. oligospora, whereas over $99 \%$ of the predicted genes in D. stenobrocha genome were located in the highly syntenic regions. Most of the predicted homologous genes were highly conserved between those two genomes. Compared with the D. stenobrocha genes in the syntenic regions, fewer than $50 \%$ of the genes in the non-syntenic regions had annotations in 

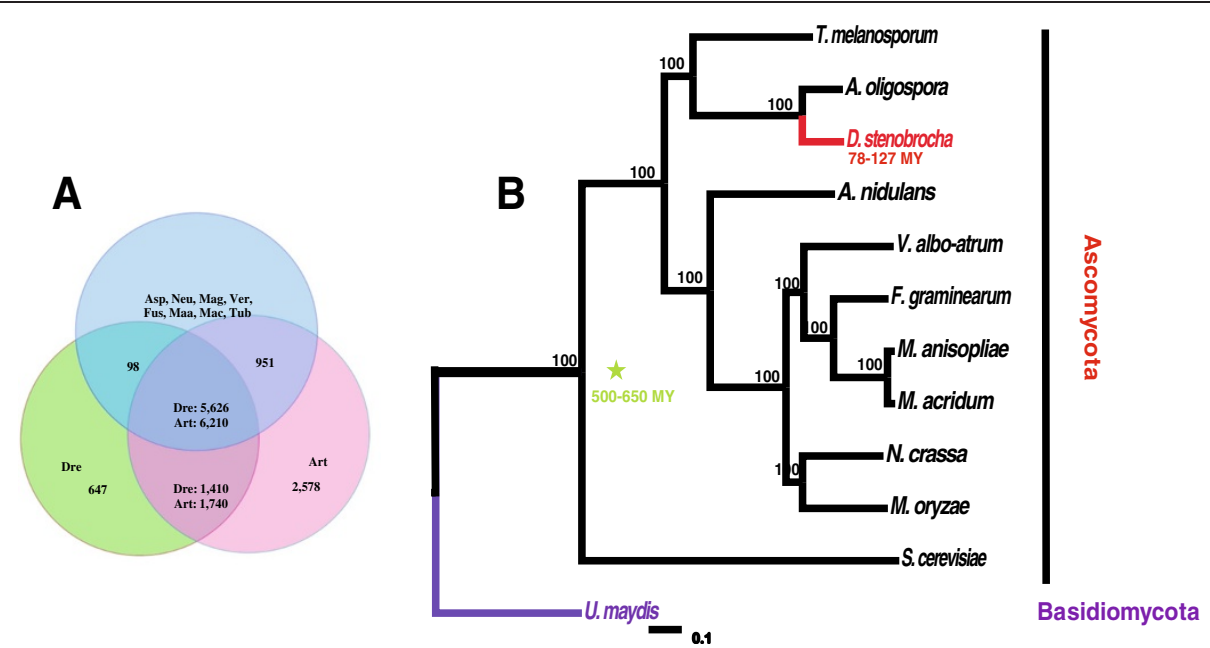

Figure 2 Homology and phylogenic relationships of $D$. stenobrocha and other selected fungi. A) Predicted proteins in Drechslerella stenobrocha (Dre) and Arthrobotrys oligospora (Art) were compared with genome coding genes in Aspergillus nidulans (Asp), Neurospora crassa (Neu), Magnaporthe oryzae (Mag), Verticillium albo-atrum (Ver), Fusarium graminearum (Fus), Metarhizium anisopliae (Maa), Metarhizium acridum (Mac) and Tuber melanosporum (Tub). B) The phylogeny tree was constructed using RAxML. Scale bar represents the number of substitutions per site. MY = million years (DRYAD accession: doi:10.5061/dryad.9n6q6; http://datadryad.org).

the Pfam database [30], indicating that the genes in nonsyntenic regions have unique gene functions. These genes may have originated because of the rich repetitive sequences and RIPs in the non-syntenic regions. Compared to the syntenic regions, 256 of the 260 transposable elements in the A. oligospora genome occurred in the nonsyntenic regions (Figure 3B), and RIPs were much more frequent in the coding regions of non-syntenic sequences. A large number of genes associated with nematode trapping, including those encoding lectins, subtilisins, chitinases, and adhesive proteins, were found in the less-syntenic and repeat-rich regions in the A. oligospora genome [22].

\section{Pathogenic functional proteins associated with} nematode-predation in $D$. stenobrocha and $A$. oligospora Chitinases and subtilisins are crucial pathogenic factors for fungal infection of both nematodes and insects. In entomopathogenic fungi such as Cordyceps militaris, $M$. anisopliae, and $M$. acridum, these enzymes can degrade insect cuticles [23,24]. The glycoside hydrolase 18 (GH18) family of chitinases restrain the growth of first-stage

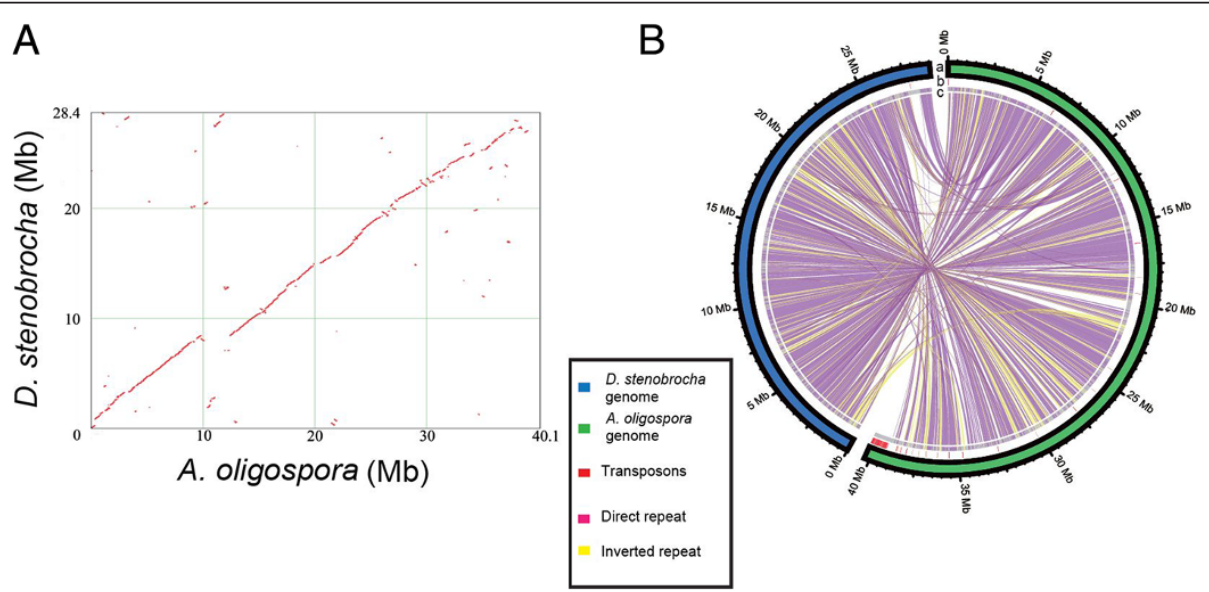

Figure 3 Genomic relationship between $D$. stenobrocha and A. oligospora. A) Syntenic relationship between A. oligospora and D. stenobrocha; B) Global view of syntenic alignments between D. stenobrocha (blue) and A. oligospora (green) and the distribution of transposable elements (TE). a: Genome sizes of the two genomes; b: Diversity of TE, red lines refer to TE; c: Grey part in the inner cycle refers to the non collinear region in the genomes; lines in the cycle indicate the syntenic region of the two genomes. 
juveniles of the nematode Meloidogyne hapla and appears to be involved in cuticle degradation during nematode infection [31,32]. A total of 17 genes encoding subtilisins were found in the $D$. stenobrocha genome, fewer than in the other genomes examined (Table 2). In contrast, the relatively high number (40) of subtilisin genes in the A. oligospora genome suggested an enhanced capacity of nematode infection. Thirteen of them were located in non-syntenic regions and shared low amino acid similarity with their homologs in the $D$. stenobrocha genome (Figure 4A). Likewise, there were eight GH18 chitinase genes in the $D$. stenobrocha genome but 15 in the A. oligospora genome; five of those genes in the $A$. oligospora genome were located in non-syntenic regions and shared low amino acid similarity with their homologs in the D. stenobrocha genome (Figure 4B). Considering $A$. oligospora as a fungal pathogen, these genes may also enable $A$. oligospora to digest the fungal cell wall and to be pathogenic to a fungus [22].

Pathogenic fungi can recognize and attach to their hosts via adhesive proteins [33,34]. Extracellular proteins from plant- and animal-pathogenic fungi that contain an eight-cysteine CFEM domain have been predicted to be involved in signal transduction or adhesion in plant hostpathogen interactions $[24,35]$. Genomic sequences revealed that there were also large numbers of CFEM-containing proteins in nematode-trapping fungi (12 in D. stenobrocha, 17 in A. oligospora), similar to insect pathogens (17 in $M$. anisopliae and 11 in M. acridum). The proteins contain a signal for secretion were presented much better in nematode-trapping fungi (11 in D. stenobrocha, 12 in $A$. oligospora) than in entomopathogens (4 in M. anisopliae, 2 in M. acridum) (Additional file 2: Table S2) [24], indicating that these genes may function in nematode trapping. Other putative adhesive proteins, such as those containing the GLEYA domain, which binds the lectin-like ligand domain [36], have also been implicated in nematode trapping. Six genes encoding GLEYA-containing proteins were identified in each nematode-trapping species (versus an average 4.5 in the entomopathogens; Additional file 2: Table S2). These adhesive proteins may play more important roles in hydrophilic soil, compared the hydrophobic phyloplane environment of entomopathogens.

\section{Weak capacity of lectin-mediated recognition in mechanical trapping}

Lectins, which are carbohydrate-binding proteins, are highly specific for their sugar moieties [37], and they are thought to be involved in fungus-nematode recognition, especially in adhesive trapping [38]. Lectin-mediated recognition has been identified in nematode-trapping fungi based on the presence of lectins associated with glycosyls of N-acetyl-D-galactosamine (GalNAc), D-glucose, D-mannose, and L-fucose [38-40] in different adhesive network-forming species. Seventeen lectin genes were predicted in A. oligospora. They mainly recognized GalNAc and fucose, e.g., four H-type lectins and three ricin B lectins recognized GalNAc, and four lectins recognized fucose (Additional file 2: Table S2). Consistent with the lectins identified from the surface of A. oligospora trapping organs [38], the presence of these GalNAc-binding lectin genes highlighted the role of GalNAc-recognition in adhesive trapping. However, the fucose-binding lectins do not match the lectins identified from traps and they should be further investigated.

Compared with $A$. oligospora, there were fewer lectin genes in the D. stenobrocha genome; only one GalNAcbinding lectin (4 in A. oligospora) and one fucose-binding

Table 2 Number of genes involved in chitin, cellulose and protein degradation of $D$. stenobrocha and selected fungi

\begin{tabular}{|c|c|c|c|c|c|c|c|c|c|}
\hline \multirow[b]{2}{*}{ Protein families } & \multirow{2}{*}{$\begin{array}{c}\text { Chitinase } \\
\text { GH18 }\end{array}$} & \multicolumn{5}{|c|}{ Cellulose degradation } & \multirow[b]{2}{*}{ CBM1 } & \multicolumn{2}{|c|}{ Protein degradation } \\
\hline & & GH5 & GH6 & GH7 & GH74 & GH61 & & A01 & S08 \\
\hline D. stenobroch $a^{a}$ & 8 & 15 & 1 & 4 & 3 & 12 & 36 & 19 & 17 \\
\hline A. oligospora & 15 & 21 & 2 & 6 & 4 & 26 & 84 & 30 & 42 \\
\hline M. acridum & 19 & 9 & 0 & 0 & 1 & 1 & 2 & 24 & 31 \\
\hline M. anisopliae & 27 & 9 & 0 & 0 & 1 & 2 & 4 & 32 & 45 \\
\hline T. melanosporum & 5 & 7 & 0 & 0 & 1 & 3 & 3 & 3 & 6 \\
\hline A. nidulans & 17 & 16 & 2 & 3 & 3 & 9 & 6 & 10 & 2 \\
\hline N. crassa & 12 & 6 & 3 & 5 & 2 & 16 & 21 & 18 & 6 \\
\hline M. oryzae & 15 & 13 & 3 & 6 & 2 & 23 & 19 & 20 & 24 \\
\hline V. albo-atrum & 13 & 13 & 4 & 6 & 4 & 21 & 22 & 16 & 20 \\
\hline F. graminearum & 18 & 14 & 1 & 2 & 2 & 14 & 12 & 18 & 26 \\
\hline
\end{tabular}

The annotation is based on CAZy classification (www.cazy.org) and MEROPS database (http://merops.sanger.ac.uk). The subfamilies are listed by the functions it involved in.

${ }^{a}$ Abbreviation for the fungal genera: Drechslerella stenobrocha, Arthrobotrys oligospora, Metarhizium acridum, Metarhizium anisopliae, Tuber melanosporum, Aspergillus nidulans, Neurospora crassa, Magnaporthe oryzae, Verticillium albo-atrum, Fusarium graminearum. 

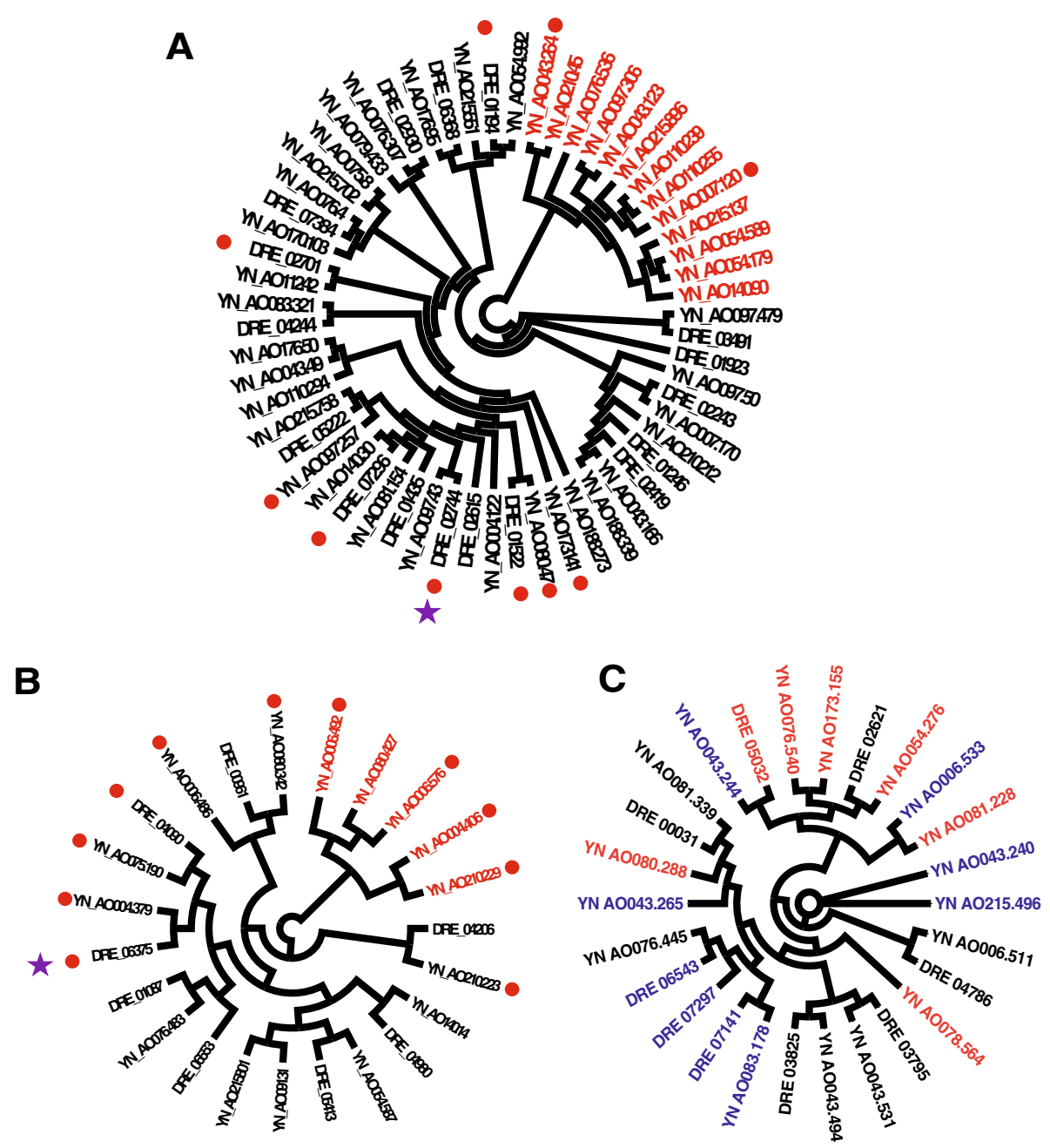

Figure 4 Unrooted phylogenetic trees showing differences in gene expansion in D. stenobrocha and A. oligospora. A) Subtilisins (peptidase S08); B) Chitinases (GH18); C) Lectins. Red branches in file $\mathbf{A}$ and $\mathbf{B}$ identify genes only present in A. oligospora genome. Red branches in file $\mathbf{C}$ identify the fungal-fucose lectin coding genes and blue branches identify GalNAc-type lectin coding genes. Secreted enzymes are marked with red circles and differently expressed genes during nematode-trapping are marked with purple stars.

lectin (5 in A. oligospora) were predicted (Additional file 2: Table S2), suggesting that D. stenobrocha had a weak capacity for lectin-mediated recognition. Given the mechanical-trapping mechanism of D. stenobrocha, lectin-mediated recognition might be functionally replaced by the actively ring constricting within a fraction of second $(0.1 \mathrm{~s})$. In addition, D. stenobrocha has a number of bulb-type lectins (Additional file 2: Table S2, Table S3), which are known to be involved in the defense of the cotton leafworm [41]. These facts suggest that $D$. stenobrocha may use lectins as a defense against nematodes rather that to adhere to them.

Lectins with the highest amino acid similarity were not classified into the same protein family, indicating multiple origins and convergent evolution (Figure 4C). Glycosyls bound by the lectins may change during fungus-nematode coevolution. These evolutionary changes could be adaptations to the prey of the adhesive-trapping fungi, although the biological significance of this coevolution is not yet clear.

Predation originated in carbon-rich environments as evidenced by $\mathrm{GH}$ families and the rampant expansion of CBM1 genes

Because nematode-trapping fungi were hypothesized to have evolved in response to carbohydrate-rich but nitro gen-poor environments [11], we examined their carbohydrate use in detail, and the numbers of carbohydrateactive enzymes (CAZymes) were analyzed for differences between nematode-trapping fungi and others [42]. Nema tode-trapping fungi had abundant glycoside hydrolases (GHs) for cellulose degradation. A total of $147 \mathrm{GH}$ genes 
in D. stenobrocha genome and 226 in A. oligospora were predicted, similar to the average number (181) in entomopathogenic fungi but fewer than in plant pathogenic (280) and saprophytic (232) fungi (Additional file 2: Table S4).

In filamentous fungi, cellulose is mainly degraded by endoglucanases and cellobiohydrolases in the cellulase families GH1, GH3, GH5, GH6, GH7, and GH61 [43-48]. The GH5 family, previously known as "cellulase family A" (http://www.cazypedia.org), were the most highly expanded GH family. Both D. stenobrocha (15) and A. oligospora (20) contained larger number genes from the GH5 family compared to other fungi except A. nidulans (16). The GH61 family was first identified in cultures of Phanerochaete carnosa performing oxidative attack of crystalline cellulose on wood [49]. The A. oligospora genome has the most GH61 genes (26), nearly twice as many as D. stenobrocha and nine times as many as entomopathogens (1.5). However, phytopathogenic fungi averaged $19 \mathrm{GH} 61 \mathrm{~s}$ and saprophytic fungi averaged 12, indicating that the cellulose-degrading capacity of nematode-trapping fungi was more like that of phytopathogens or saprobes than entomopathogens. This may also be supported by the niches where enriched in the cellulose materials (e.g. leaves) for trapping fungi and where was less cellulose materials (stem of plants and phyloplane) for entomopathogens. In addition, the GH7 family was well represented in nematode-trapping fungi (6 in A. oligospora, 4 in $D$. stenobrocha) compared with other fungi (average 3 ). The GH74 family, which encodes xyloglucans/xyloglucanoligosaccharides, are also presented better in nematodetrapping fungi (3 in D. stenobrocha, 4 in A. oligospora) than other compared fungi (average 2).

Phytopathogens in particular have additional GH3, GH11, and other GH families classified in the pathogenhost interaction (PHI) database. The two nematode-trap ping fungi (averaged 8 GH3 and 2 GH11 genes) had fewer than in phytopathogenic fungi (averaged $20 \mathrm{GH} 3$ and 4 GH11 genes). The contraction of these genes associated with plant-infection might explain why nematode-trapping fungi contain fewer total GHs but have a similar capacity for cellulose degradation.

The activity of CAZy enzymes can be enhanced by carbohydrate-binding module 1 (CBM1) protein domains within these proteins [50]. These domains may increase enzyme efficiency by enhancing the localization of enzymes on the surface of crystalline cellulose [50] or disturb the structure of crystalline cellulose [51]. There were 37 CBM1-containing genes in the D. stenobrocha genome, half the number (86) in A. oligospora but three to four times as many as in the other fungal genomes (average 12, Table 2). Twenty-six of the 37 D. stenobrocha CBM1containing proteins had a signal peptide for secretion, indicating that these enzymes function extracellularly. The typical saprophytic white rot fungus (Phanerochaete chrysosporium) and the coprophilous Podospora anserina had the most CBM1-containing protein genes [52,53]. There were 19 GHs containing CBM1 domains in D. stenobrocha and 47 in A. oligospora, compared with 16 in $P$. anserina (Figure 5). However, CBM1-containing polysaccharide lyases which were specific to plant pathogens and were absent in nematode-trapping and saprophytic fungi [25]. Thus, the CBM1 profiles of nematode-trapping fungi were similar to those of saprophytic fungi but different from those of plant pathogenic fungi.

The capacities of secreted enzymes to degrade carbohydrates and nitrogenous compounds might also be expected to differ among fungi, and this might be reflected in the genes related to their secretomes. Therefore, the secretomes of $D$. stenobrocha and $A$. oligospora were compared with those of entomopathogens to identify enzymes involved in carbon and nitrogen resource utilization. $A$. oligospora had a large number of secreted proteins (646) compared with entomopathogens (406 in M. acridum, 546 in M. anisopliae), and twice as many as D. stenobrocha (355) (Additional file 2: Table S5). The GH proteins in the nematode-trapping fungi secretomes showed a completely different profile from those in entomopathogens. Carbohydrate use was enhanced in nematode-trapping fungi, while protein degradation was weakened. Indeed, carbohydrate-degrading enzymes were much more likely to be shared between nematode-trapping species (22\% shared) than were proteases $(5-6 \%)$, as opposed to in entomopathogens $(9-10 \%$ and $12-14 \%$ shared, respectively) (Additional file 1: Figure S2). Thus the large number of predicted genes for carbohydrate-degradation and the reduced number for nitrogen resource use supported the hypothesis that nematode-trapping fungi originated in carbon-rich but nitrogen-poor environments.

\section{Transcriptional responses during nematode trap formation reveal clues to the transition from saprophagy to predation}

To identify the putative signal and metabolic pathways involved in trap formation and the nematode trapping process, we used RNA-Seq to examine the transcriptional responses of $D$. stenobrocha during the nematode-fungus interaction (GEO accession: GSE54423; www.ncbi.nlm.nih. gov/geo). Three stages the interaction were examined: (1) D. stenobrocha cultures on CMA medium free of nematodes; (2) cultures with a large number of traps $22 \mathrm{~h}$ after nematode challenge; and (3) cultures in which most of the nematodes were trapped $28 \mathrm{~h}$ after nematode challenge [54]. After sequencing over 1 million tags for each treatment, $97.2 \%$ of the genes predicted in the D. stenobrocha genome were expressed in the cultures free of nematodes. After nematode challenge for 22 and 28 h, 27\% of the predicted genes were differently expressed (probability $\geq 0.5$, NOIseq; http://bioinfo.cipf.es/noiseq) compared 
A

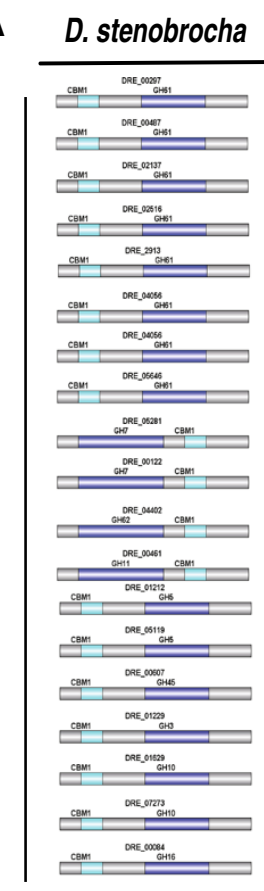

P. anserina

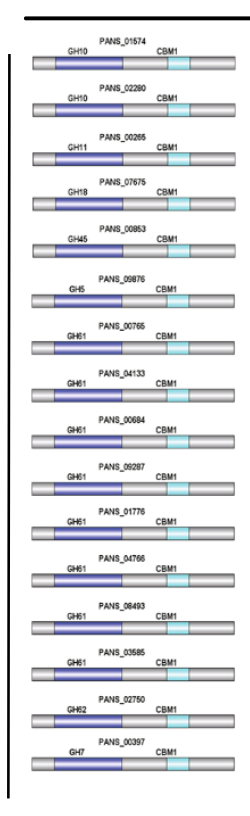

\section{A. oligospora}

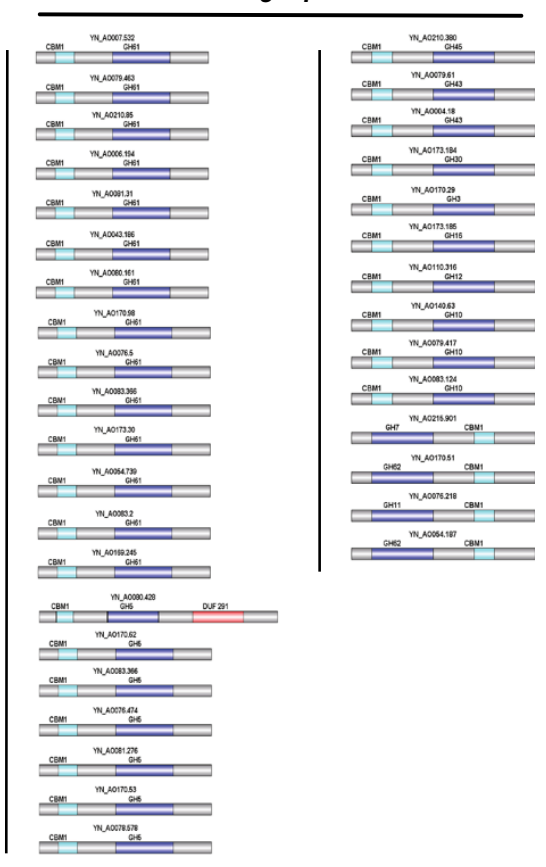

B

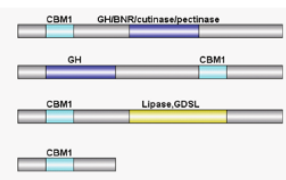

Figure 5 CBM1 appended genes in D. stenobrocha genome compared with that of $A$. oligospora and $P$. anserina. A) Glycoside hydrolases coding genes containing CBM1 domains in D. stenobrocha compared with that of $A$. oligospora and $P$. anserina, showing the expanded CBM1 appended glycoside hydrolases in nematode-trapping fungi and the different gene structures between nematode-trapping fungi and saprophytic fungi. B) Structures of CBM1 domain appended genes in D. stenobrocha genome. CBM1 + glycoside hydrolase; glycoside hydrolase + CBM1; CBM1 + lipase; CBM1.

to the nematode-free culture, including 800 that were upregulated and 1,300 that were down-regulated.

Transcripts were classified into eight functional clusters (maSigPro) to provide a global view of the transcriptional responses to nematode stimulation and trapping (Figure 6a). In general, the most significantly up-regulated genes were involved in rapid cell growth, intracellular signal transduction, and protein degradation, concomitant with the formation of trapping organs (Figure 6b). Of the 100 most highly-expressed genes during the development of the constricting rings $(22 \mathrm{~h}), 35$ were functionally uncharacterized (Additional file 2: Table S6), suggesting that several genes with unknown function were involved in the development of trapping organs. Additionally, 27 of these 35 genes were down-regulated to very low levels $28 \mathrm{~h}$ after nematode challenge (Additional file 2: Table S6). These genes should be identified to provide insight into trap formation.

Although the predatory lifestyle and constricting rings are unique to $D$. stenobrocha, during nematode trapping, signal transduction pathways activated were similar to those employed by entomopathogens during insect infection. For instance, the protein kinase $\mathrm{C}$ (PKC) signal transduction pathway is important for fungal infection of insects [23], and the up-regulation of protein kinase C (DRE_04067) also suggest a role in nematode trapping. In addition, the highly expressed G-protein alpha subunit (DRE_07451) might be the first step in the PKC pathway, activated by a protein signal from nematodes (Additional file 2: Table S7). Unexpectedly, pheromone receptors involved in fungal fruiting body formation and the sexual phase of the cycle [55] have been reported to be involved in nematode trapping in A. oligospora, but their orthologs (DRE_04115, DRE_03014 ) in D. stenobrocha were not transcribed or were transcribed at low levels during the capture of nematodes (Additional file 2: Table S7). Taken together, these data on pheromone receptor and signal transduction genes suggested that trap-formation of D. stenobrocha was more dependent on the PKC pathway than the pheromonedependent signal pathway. 


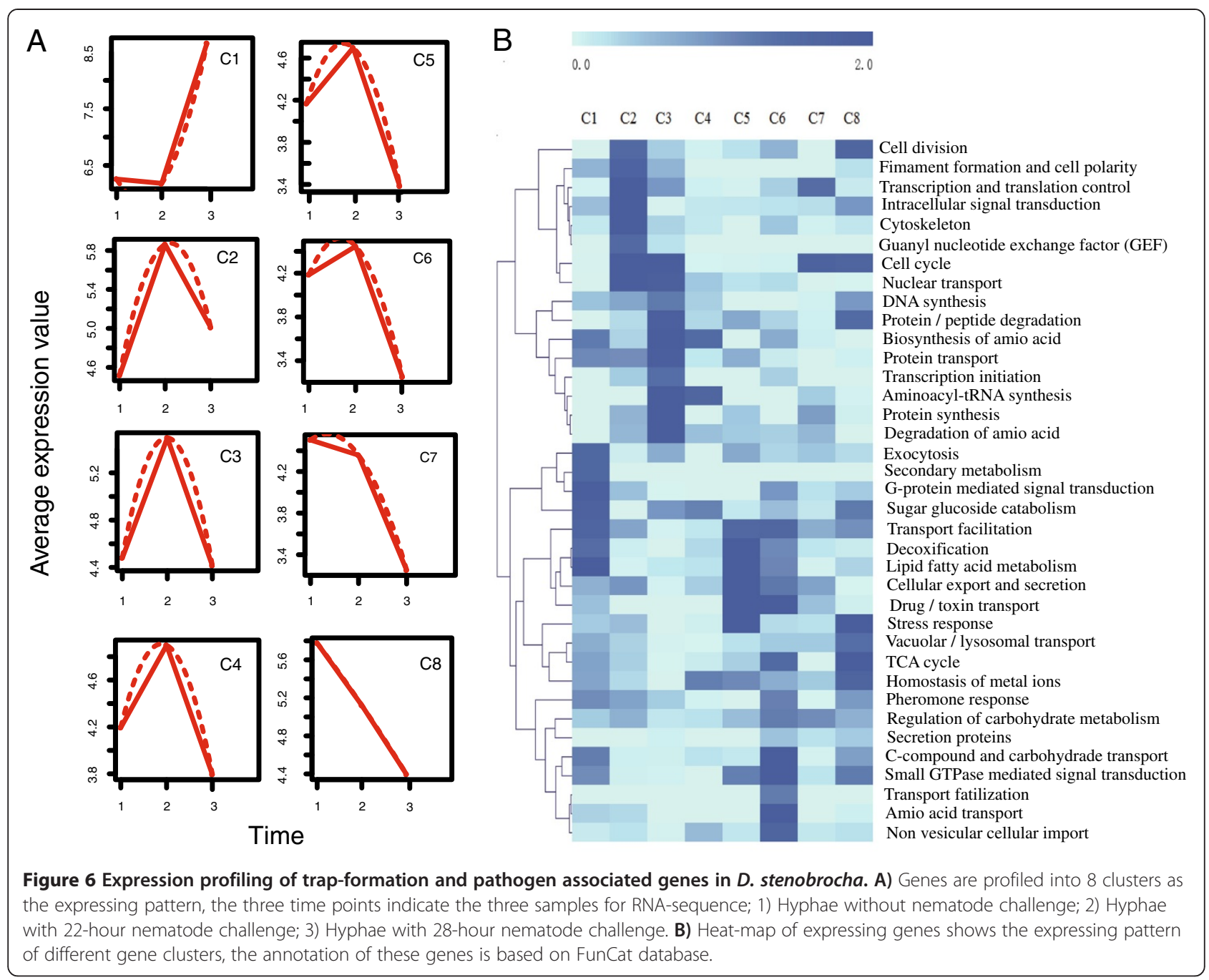

Following PKC-mediated signal transduction, transcription factors were activated to regulate the downstream genetic responses. $\mathrm{Zn}(2)-\mathrm{C} 6$ type transcription factors were predicted to be involved in the regulation of aspects of both primary and secondary metabolism in Aspergillum, including protein and carbohydrate degradation [56]. The levels of these transcription factors, coincident with the upregulation of proteases are predicted to be involved in the transition in $D$. stenobrocha from a saprophagous to a predatory stage.

The formation of constricting ring was expected to be significantly involved in the cell division. A cell division protein (DRE_05065) and a cyclin (DRE_07217) were highly expressed during the trap-formation (Additional file 2: Table S7). This indicates that these two cycle proteins may be the key genes that initiate the cell division for trapformation. Furthermore, a large number of transporters, including six genes belong to MFS (major facility superfamily) among the 100 most highly up-regulated genes, were employed by $D$. stenobrocha to utilize the nutrition of nematodes (Additional file 2: Table S6).

Differential expression of secreted enzymes revealed the distinct transition from saprophagy to predation in D. stenobrocha during the nematode-trapping process. The down-regulation of enzymes involved in carbohydrate degradation, including cellulases (GH5, GH7, and GH61) and endoxylanase (GH10), indicated the reduced importance of carbohydrate use from the substrate during nematode trapping. In contrast, the secreted enzymes involved in cuticle degradation and infection, such as chitinases (GH18) and subtilisins (peptidase S8A), were upregulated, suggesting a shift to predation. In addition, other putative serine proteases involved in nematode infection were significantly up-regulated (Table S8). These results support the hypothesis that nematode-trapping fungi are both efficient carbohydrate utilizing saprobes and nematode-trapping predators. In addition, other putative serine proteases (peptidases S28 and S33) involved in 
nematode infection were significantly up-regulated. The transcriptional profiles revealed well the transition from saprophagy to predation in nematode-trapping fungi.

\section{Discussion}

Fungal predation, especially the evolutionary origins of nematode trapping fungi and their mechanical trapping mechanism, have long attracted the interest of mycologists and other biologists. The three cells of the constricting ring rapidly triple their volumes to firmly lasso their nematode prey, but this fascinating biological phenomenon is still poorly understood. This analysis of the genome and transcriptome of $D$. stenobrocha in comparison with the genome of the adhesive network-forming A. oligospora provides insights into the origins of fungal predation, the shift from a saprophagous to a predatory stage, and molecular mechanisms of nematode trapping.

Whole genomic sequences indicated that $D$. stenobrocha is compact, showing rare RIPs and transposons. The gene NCU02034 in N. crassa, which is only known to be required for RIP [27], was absent from the D. stenobrocha genome, indicating that it lacks the ability for RIPs. The rate of gene duplication is decreased by RIP [57], implying that genomes with high frequency of RIPs generally have low gene duplication rates. D. stenobrocha is an exception. The paucity of RIP and transposons in the D. stenobrocha genome indicated that it was compact with fewer orphan genes arose compared with that of A. oligospora; only 7,781 genes were predicted. Given the function of RIPs in meiosis [27], more studies should be conducted to investigate whether A. oligospora or D. stenobrocha undergo sexual stages.

Previous studies showed that fungal recognition of the nematode was mediated by lectins on the trap surface [38]. Thus far, six kinds of lectins that recognize different glycosyls have been identified; those glycosyls are GalNAc in A. oligospora, D-glucose in Arthrobotrys conoides, L-fucose in Monacrosporium eudermatum, 2-deoxyD-glucose (2-DG) in Monacrosporium rutgerienses, sialic acid in Drechmeria coniospora, and N-acetylneuraminic in Verticillium balanoides [38-40,58]. The presence of lectins that bind GalNAc and L-fucose in A. oligospora partially supported the role of lectins in host-pathogen recognition. However, the presence of fewer lectins in the D. stenobrocha genome suggested that the mechanical-trapping mechanism might not involve lectin in the fungal recognition of nematodes. Adhesive proteins are involved in host recognition by plant and insect fungal pathogens $[33,34]$. The occurrence of adhesive proteins in two species of trapping fungi suggested that they may also play crucial roles in both mechanical and adhesive trapping.

Plant residues in forests generally result in an extremely high carbon-to-nitrogen ratio. Some wood-decaying fungi may have evolved to capture tiny animals for nitrogen under this environmental selection pressure [11]. A phylogenetic analysis based on the conservative genes of nematode-trapping fungi belonging to Ascomycetes also suggests a possible causal relationship between mass extinction events and the evolution of fungal predation [9]. Predatory fungi gained a competitive advantage over strict saprobes by predating tiny animals when available organic matter decreased during the ecosystem recovery [9]. Although this hypothesis lacks direct evidence, comparative analyses of the genome of $D$. stenobrocha provide support for this hypothesis.

In this study, comparison of the selected genomes revealed that genes involved in cellulolytic degradation were abundant in nematode-trapping fungi, whereas genes for enzymes that degrade living plant cells were rare, indicating that nematode-trapping fungi share more similarities with saprobes than with phytopathogens. The divergence time of the adhesive-trapping fungus, A. oligospora with greater saprophytic capacity, was much later than that of D. stenobrocha [9]. Our hypothesis that nematode-trapping fungi originated from efficient cellulolytic fungi was partially supported by evidence that constricting ring-trapping fungi with weaker saprophytic capacity evolved to capture nematodes after the mass extinction events of the Permian-Triassic (251.4 MY), whereas adhesive-trapping fungi survived the Permian-Triassic boundary with the enhanced saprophytic capacity and evolved to capture nematodes after the mass extinction of the Triassic-Jurassic. More genomes of nematode-trapping fungi with different trapping organs should be analyzed.

The density dependence of nematophagous fungi also varies with the availability of saprophytic component. Adhesive net-forming $A$. oligospora has higher diversity in the nutrition poor niches indicating its higher saprophytic capacity [14]; while constricting ring-forming $D$. stenobrocha always exists in the nutrition rich niches suggesting its weaker saprophytic capacity than A. oligospora [14]. The enhanced saprophytic capacity of A. oligospora was evidenced by the greater expansion of CBM1-containing GHs, which revealed that A. oligospora depends more on saprophagy than does $D$. stenobrocha, suggesting that $A$. oligospora is more competitive when nematodes are absent, in addition $D$. stenobrocha might be more dependent on nematodes for nutrition. The dependence of D. stenobrocha on nematodes might explain why its constricting rings can form spontaneously, whereas the network of $A$. oligospora must always be induced.

Although the genome sequence of $D$. stenobrocha does not provide specific evidence about ring-cell inflation nor ring constriction, a number of orphan genes in the genome may contribute specifically to this process. Among the 470 expressed orphan genes, more than 120 were differently expressed in the presence of nematodes, suggesting that they encode proteins that are involved in ring 
constriction. However, more than 90 of these orphan genes were not annotated in the Pfam database, including a large number of those that were most significantly upregulated. These uncharacterized genes that were expressed during ring constriction imply that a novel mechanism is involved. These genes provide candidates to investigate in future functional studies.

\section{Conclusion}

In conclusion, the rare occurrence of transposons and RIPs indicated the slow evolution and primitive state of the D. stenobrocha genome. The similarity of carbohydratedegrading enzyme catalogs between trapping fungi and efficient cellulolytic fungi support the saprophytic origin of predation. The down-regulation of saprophagy-related genes and the up-regulation of predation-related ones during nematode infection revealed the transition between dual strategies of saprophagy and predation in this trapping fungus. The reduced number of lectin genes in $D$. stenobrocha did not support lectin-mediated recognition in mechanical trapping. The high expression levels of uncharacterized orphan genes imply that an unknown mechanism is involved in ring constriction. Overall, the genomic and transcriptome sequences of D. stenobrocha provided new insight into the origin of nematode predation in fungi and the mechanism of constricting rings, these sequences also can be essential tools to reveal the mechanism of nematode-capture by constricting rings, and for further better exploitation of nematode-trapping fungi in the use of nematode control.

\section{Methods}

\section{Fungal strains}

D. stenobrocha strain (CGMCC 3.6768) was selected for genome sequencing and has been studied well in the laboratory which can be cultured on artificial medium such as PDA and CMA plates and maintained on PDA Plate. D. stenobrocha spores were cultured on CMA plate for 4 days and challenged with 500 nematodes each plate. Samples were prepared [59] and structures were observed by scanning electron microscope Quanta200, produced by FEI. This fungus was originally isolated by Drechsler in 1937 and we isolated from the soil of Yunnan province in 2004. It provides important materials to research the mechanisms of constricting rings.

\section{Genome sequencing and assembly}

The genome of $D$. stenobrocha was sequenced by shotgun using a Solexa system for massively parallel pyrosequencing at BGI (Shenzhen, China). This resulted in 4,299 Mb of sequence data $(80 \times$ coverage $)$ with the useful length of 2,317 Mb. By using the SOAPdenovo software [60], assembly was performed producing 142 contigs and reached the size of $29.02 \mathrm{Mb}$.

\section{Gene prediction and annotation}

In order to reach high accuracy, the gene structures of D. stenobrocha were predicted with EVidenceModeler (EVM) [61] algorithms and the sequenced Fusarium graminearum was used as a reference. PseudoPipe was selected with default settings conducting the pseudogene identification [62]. Finally, the prediction was performed by Blast against protein database and InterProscan searches against protein domain databases. Potential secreted proteins of $D$. stenobrocha and other species were predicted and compared by SignalP 3.0 [63] analysis using Hidden Markov model.

\section{Orthology and phylogenomic analysis}

Predicted proteins of $D$. stenobrocha were compared with the predicted proteins of other sequenced 9 fungi. The comparison of all proteins was performed by BLASTP against all the other proteins in these genomes. Sequences with $\mathrm{E} \leq 1 \mathrm{e}^{-5}$ and as least $40 \%$ sequence identity over $60 \%$ were considered homologous sequences. Totally 1441 ortholog genes were acquired and aligned with MAFFT [64]. Amino acid sequences were used by the program RAxML to create a maximum likelihood tree [65]. Divergence time between the compared species was estimated by PL method [66] with r8s version 1.8 (http://loco.biosci. arizona.edu/r8s/) using the calibration against the origin of Ascomycota at 500-600 million years ago [67].

\section{Protein family classification and evolution analysis}

Protein families of the whole genome were classified by use of InterproScan analysis in order to identify genes descended from a common ancestor [68]. Putative enzymes involved in carbohydrate utilization were identified by blast searching against carbohydrate-active enzymes database (http://www.cazy.org/). Protease protein families were classified by blast against MEROPS database. And additionally, G-protein coupled receptors, protein kinases, transcription factors were identified by the significant sequence of GPCDB 7 transmembrane helices (http://www.cbs.dtu.dk/ services/TMHMM/), KinBase (http://kinase.com/) and fungal transcription database (http://ftfd.snu.ac.kr/). The evolution of the protein families' size and knot point was analyzed by CAFÉ [69].

Repeat and Repeat-induced point mutation (RIP) analysis Genomic repetitive analyses were performed by Blasted against the PILER [70], PepeatMasker library [71] and TRF [72]. RIP index was determined by program RIPCAL [26].

\section{Transcriptome analysis}

Caenorhabditis elegans were cultured in NGM manual liquid medium for 4 days, and collected by $0.01 \mathrm{~mm}$ filter membrane. The Escherichia coli strain, OP50, was 
cultured in LB medium for food of nematodes [73]. The spores of $D$. stenobrocha with the number of $5.2 \times 10^{5}$ were cultured in 100-mL PDB liquid medium for four days. Hyphae was collected by glass cotton felt and washed with water three times. Hyphae from each 100$\mathrm{mL}$ liquid medium were left on two CMA medium plate for two days and co-cultured with 2500 C. elegans each plate. After 22- and 28-hours challenge at $25^{\circ} \mathrm{C}$, RNA and was exacted by the method of TRizol [74]. Functions of the expressed genes were predicted based on FunCat database. Program maSigPro was performed to enrich the genes expressed in different patterns [75]. Probability value of each differently expressed gene is calculated by program NOISeq [76].

\section{Additional files}

Additional file 1: Figure S1. Distribution of paralogous gene numbers with different levels of nucleotide similarity in D. stenobrocha and other fungi. Figure S2. Classification and comparison of secreted proteins by function of nematode-trapping fungi (D. stenobrocha, A. oligospora) and insect pathogens (M. acridum, M. anisopliae). Each circle represents fraction of genes in each genome, and the percentages are also shown.

Additional file 2: Table S1. Genome sizes and numbers of protein coding genes in Drechslerella stenobrocha and other fungi. Table S2. Abundance of lectins and adhesive proteins in Drechslerella stenobrocha, Arthrobotrys oligospora, and other fungi. Table S3. Predicted lectin coding genes in Drechslerella stenobrocha and Arthrobotrys oligospora genomes. Table S4. Carbohydrate-degrading enzymes in Drechslerella stenobrocha and other fungi arranged by glycoside hydrolase family. Table S5. Numbers of secreted proteins in the Drechslerella stenobrocha and other fungal genomes. Table S6. The 100 most differentially-expressed genes in Drechslerella stenobrocha while trapping nematodes. Table S7. Transcriptional response of genes involved in signal transduction of Drechslerella stenobrocha. Table S8. Expression of secreted enzymes in the three transcriptomes of Drechslerella stenobrocha.

\section{Abbreviations \\ 2-DG: 2-deoxy-D-glucose; CAMP: Cyclic adenosine monophosphate; CAZyme: Carbohydrate-active enzymes; CBM1: Carbohydrate-binding module 1; CDC: Cell division cycle; GalNAc: N-acetyl-D-galactosamine; MFS: Major facility superfamily; MY: Million years; GH: Glycoside hydrolase; IP3: Inositol-1, 4, 5-triphosphate; PHI: Pathogen-host interaction; PKC: Protein kinase C; RIP: Repeat-induced point.}

\section{Competing interests}

The authors declare that they have no competing interests.

\section{Authors' contributions}

$\mathrm{KL}$ conducted the molecular experiments. $\mathrm{XZ}$ assembled the genome and annotated the genes. WZ analyzed the data and wrote the manuscript. $Y L$ and XW helped to analyze the data. MX helped with the molecular experiments. XL designed the study and wrote the paper. All authors have read and approved the manuscript.

\section{Acknowledgements}

The authors thank Dr. Leah Larkin at University of New Mexico for suggestion and editing for the manuscript, Chengshu Wang at Institute of Plant Physiology and Ecology, SIBS, CAS for the valuable suggestion and discussion.

\section{Author details}

'State Key Laboratory of Mycology, Institute of Microbiology, Chinese Academy of Sciences, No 3 1st Beichen West Rd., Chaoyang District, Beijing 100101, China. ${ }^{2}$ University of Chinese Academy of Sciences, Beijing 100039, China.
Received: 18 September 2013 Accepted: 4 February 2014

Published: 8 February 2014

\section{References}

1. Barron GL: The Nematode-destroying Fungi. Guelph: Canadian Biological Publications; 1977 .

2. Gray N: Ecology of nematophagous fungi: distribution and habitat. Ann Appl Biol 1983, 102:501-509.

3. Ahrén D, Tholander M, Fekete C, Rajashekar B, Friman E, Johansson T, Tunlid A: Comparison of gene expression in trap cells and vegetative hyphae of the nematophagous fungus Monacrosporium haptotylum. Microbiology 2005, 151:789-803.

4. Liu K, Tian J, Xiang M, Liu X: How carnivorous fungi use three-celled constricting rings to trap nematodes. Protein cell 2012, 3:325-328.

5. Nordbring-Hertz B, Jansson HB, Tunlid A: Nematophagous Fungi. Chichester: John Wiley \& Sons, Ltd; 2006.

6. Yang $Y$, Yang E, An Z, Liu X: Evolution of nematode-trapping cells of predatory fungi of the Orbiliaceae based on evidence from rRNA-encoding DNA and multiprotein sequences. Proc Natl Acad Sci U S A 2007, 104:8379-8384.

7. Larsen M: Prospects for controlling animal parasitic nematodes by predacious micro fungi. Parasitology 2000, 120:121-131.

8. Kerry B: Rhizosphere interactions and the exploitation of microbial agents for the biological control of plant-parasitic nematodes. Annu Rev Phytopath 2000, 38:423-441.

9. Yang E, Xu L, Yang Y, Zhang X, Xiang M, Wang C, An Z, Liu X: Origin and evolution of carnivorism in the Ascomycota (fungi). Proc Natl Acad Sci U S A 2012, 109:10960-10965.

10. Schmidt AR, Dörfelt H, Perrichot V: Carnivorous fungi from Cretaceous amber. Science 2007, 318:1743.

11. Barron GL: Predatory fungi, wood decay, and the carbon cycle. Biogeosciences 2003, 4:3-9.

12. Pramer D: Nematode-trapping fungi. Science 1964, 144:382-388.

13. Gray N: Nematophagous fungi with particular reference to their ecology. Biol Rev 1987, 62:245-304.

14. Gray N: Ecology of nematophagous fungi: effect of soil moisture, organic matter, $\mathrm{pH}$ and nematode density on distribution. Soil Bio Bioch 1985, 17:499-507.

15. Linford MB: Stimulated activity of natural enemies of nematodes. Science 1937, 85:123-124.

16. Nordbring-Hertz B: Nematode-induced morphogenesis in the predacious fungus Arthrobotrys oligospora. Nematologica 1977, 23:443-451.

17. Drechsler C: Some hyphomycetes that prey on free-living terricolous nematodes. Mycologia 1937, 29:447-552.

18. Chen TH, Hsu CS, Tsai PJ, Ho YF, Lin NS: Heterotrimeric G-protein and signal transduction in the nematode-trapping fungus Arthrobotrys dactyloides. Planta 2001, 212:858-863.

19. Muller $\mathrm{H}$ : The constricting ring mechanism of two predacious hyphomycetes. T Brit Mycol Soc 1958, 41:341-364.

20. Niu XM, Zhang KQ: Arthrobotrys oligospora: a model organism for understanding the interaction between fungi and nematodes. Mycologia 2011, 2:59-78

21. Lopez-Llorca LV, Jansson HB, Macia V, Salinas J: Nematophagous fungi as root endophytes. In Microbial Root Endophytes, Volume 11. Berlin: Springer Berlin Heidelberg; 2006:191-206.

22. Yang J, Wang L, Ji X, Feng Y, Li X, Zou C, Xu J, Ren Y, Mi Q, Wu J: Genomic and proteomic analyses of the fungus Arthrobotrys oligospora provide insights into nematode-trap formation. PLoS Pathog 2011, 7:e1002179.

23. Zheng P, Xia Y, Xiao G, Xiong C, Hu X, Zhang S, Zheng H, Huang Y, Zhou Y, Wang S: Genome sequence of the insect pathogenic fungus Cordyceps militaris, a valued traditional Chinese medicine. Genome Biol 2011, 12:R116.

24. Gao Q, Jin K, Ying SH, Zhang Y, Xiao G, Shang Y, Duan Z, Hu X, Xie XQ, Zhou G: Genome sequencing and comparative transcriptomics of the model entomopathogenic fungi Metarhizium anisopliae and M. acridum. PLoS Genet 2011, 7:e1001264.

25. Klosterman SJ, Subbarao KV, Kang S, Veronese P, Gold SE, Thomma BP, Chen Z, Henrissat B, Lee YH, Park J: Comparative genomics yields insights into niche adaptation of plant vascular wilt pathogens. PLoS Pathog 2011, 7:e1002137.

26. Hane JK, Oliver RP: RIPCAL: a tool for alignment-based analysis of repeatinduced point mutations in fungal genomic sequences. $B M C$ Bioinformatics 2008, 9:478. 
27. Freitag M, Williams RL, Kothe GO, Selker EU: A cytosine methyltransferase homologue is essential for repeat-induced point mutation in Neurospora crassa. Proc Natl Acad Sci U S A 2002, 99:8802-8807.

28. Tyler BM, Tripathy S, Zhang X, et al: Phytophthora genome sequences uncover evolutionary origins and mechanisms of pathogenesis. Science 2006, 313:1261-1266.

29. Goodwin SB, M'Barek SB, Dhillon B, Wittenberg AH, Crane CF, Hane JK, Foster AJ, Van der Lee TA, Grimwood J, Aerts A: Finished genome of the fungal wheat pathogen Mycosphaerella graminicola reveals dispensome structure, chromosome plasticity, and stealth pathogenesis. PLOS Genet 2011, 7:e1002070.

30. Finn RD, Tate J, Mistry J, Coggill PC, Sammut SJ, Hotz H-R, Ceric G, Forslund K, Eddy SR, Sonnhammer EL: The Pfam protein families database. Nucleic Acids Res 2008, 36(suppl 1):D281-D288.

31. Fan Y, Fang W, Guo S, Pei X, Zhang Y, Xiao Y, Li D, Jin K, Bidochka MJ, Pei Y: Increased insect virulence in Beauveria bassiana strains overexpressing an engineered chitinase. Appl Environ Microbiol 2007, 73:295-302.

32. Mercer C, Greenwood D, Grant J: Effect of plant and microbial chitinases on the eggs and juveniles of Meloidogyne hapla Chitwood (Nematoda: Tylenchida). Nematologica 1992, 1:227-236.

33. Cormack BP, Ghori N, Falkow S: An adhesin of the yeast pathogen Candida glabrata mediating adherence to human epithelial cells. Science 1999, 285:578-582.

34. Staab JF, Bradway SD, Fidel PL, Sundstrom P: Adhesive and mammalian transglutaminase substrate properties of Candida albicans Hwp1. Science 1999, 283:1535-1538.

35. Kulkarni RD, Thon MR, Pan H, Dean RA: Novel G-protein-coupled receptor-like proteins in the plant pathogenic fungus Magnaporthe grisea. Genome Biol 2005, 6:R24.

36. Linder T, Gustafsson CM: Molecular phylogenetics of ascomycotal adhesins - a novel family of putative cell-surface adhesive proteins in fission yeasts. Fungal Genet Biol 2008, 45(4):485-497.

37. Sharon N, Lis H: Lectins as cell recognition molecules. Science 1989, 246:227

38. Nordbring-Hertz B, Mattiasson B: Action of a nematode-trapping fungus shows lectin-mediated host-microorganism interaction. Nature 1979, 281:477-479.

39. Rosenzweig W, Ackroyd D: Binding characteristics of lectins involved in the trapping of nematodes by fungi. App/ Environ Microbiol 1983, 46:1093-1096

40. Nordbring-Hertz B: Dialysis membrane technique for studying microbial interaction. Appl Environ Microbiol 1983, 45:290-293.

41. Sadeghi A, Smagghe G, Broeders S, Hernalsteens J-P, De Greve H, Peumans WJ, Van Damme EJ: Ectopically expressed leaf and bulb lectins from garlic (Allium sativum L.) protect transgenic tobacco plants against cotton leafworm (Spodoptera littoralis). Transgenic Res 2008, 17:9-18.

42. Cantarel BL, Coutinho PM, Rancurel C, Bernard T, Lombard V, Henrissat B: The Carbohydrate-Active EnZymes database (CAZy): an expert resource for glycogenomics. Nucleic Acids Res 2009, 37(suppl 1):D233-D238.

43. Gielkens MM, Dekkers E, Visser J, De Graaff LH: Two cellobiohydrolaseencoding genes from Aspergillus niger require D-xylose and the xylanolytic transcriptional activator XInR for their expression. Appl Environ Microbiol 1999, 65:4340-4345.

44. Grassick A, Murray PG, Thompson R, Collins CM, Byrnes L, Birrane G, Higgins TM, Tuohy MG: Three-dimensional structure of a thermostable native cellobiohydrolase, $\mathrm{CBH} \mathrm{IB}$, and molecular characterization of the cel7 gene from the filamentous fungus, Talaromyces emersonii. Eur J Biochem 2004, 271:4495-4506.

45. Liu Y, Yoshida M, Kurakata Y, Miyazaki T, Igarashi K, Samejima M, Fukuda K, Nishikawa A, Tonozuka T: Crystal structure of a glycoside hydrolase family 6 enzyme, CcCel6C, a cellulase constitutively produced by Coprinopsis cinerea. FEBS J 2010, 277:1532-1542.

46. Cohen R, Suzuki MR, Hammel KE: Processive endoglucanase active in crystalline cellulose hydrolysis by the brown rot basidiomycete Gloeophyllum trabeum. Appl Environ Microbiol 2005, 71:2412-2417.

47. Toda H, Takada S, Oda M, Amano Y, Kanda T, Okazaki M, Shimosaka M: Gene cloning of an endoglucanase from the basidiomycete Irpex lacteus and its CDNA expression in Saccharomyces cerevisiae. Biosci Biotech Bioch 2005, 69:1262-1269.

48. Bastien L, Sawyer N, Grygorczyk R, Metters KM, Adam M: Cloning, functional expression, and characterization of the human prostaglandin E2 receptor EP2 subtype. J Biol Chem 1994, 269:11873-11877.
49. MacDonald J, Doering M, Canam T, Gong Y, Guttman DS, Campbell MM, Master ER: Transcriptomic responses of the softwood-degrading white-rot fungus Phanerochaete carnosa during growth on coniferous and deciduous wood. Appl Environ Microbiol 2011, 77:3211-3218.

50. Guillén D, Sánchez S, Rodríguez-Sanoja R: Carbohydrate-binding domains: multiplicity of biological roles. Appl Microbiol Biotech 2010, 85:1241-1249.

51. Wang $L$, Zhang $Y$, Gao $P$ : A novel function for the cellulose binding module of cellobiohydrolase I. Sci China Life Sci 2008, 51:620-629.

52. Espagne E, Lespinet $O$, Malagnac F, Da Silva C, Jaillon O, Porcel BM, Couloux A, Aury J-M, Ségurens B, Poulain J: The genome sequence of the model ascomycete fungus Podospora anserina. Genome Biol 2008, 9:R77.

53. Floudas D, Binder M, Riley R, Barry K, Blanchette RA, Henrissat B, Martínez AT, Otillar R, Spatafora JW, Yadav JS: The Paleozoic origin of enzymatic lignin decomposition reconstructed from 31 fungal genomes. Science 2012, 336:1715-1719.

54. Xie H, Aminuzzaman F, Xu L, Lai Y, Li F, et al: Trap induction and trapping in eight nematode-trapping fungi (Orbiliaceae) as affected by juvenile stage of Caenorhabditis elegans. MYCOPATHOLOGIA 2010, 169:467-473.

55. Pöggeler S, Nowrousian M, Ringelberg C, Loros J, Dunlap J, Kück U: Microarray and real-time PCR analyses reveal mating type-dependent gene expression in a homothallic fungus. Mol Genet Genomics 2006, 275:492-503.

56. Pel HJ, de Winde JH, Archer DB, Dyer PS, Hofmann G, Schaap PJ, Turner G, de Vries RP, Albang R, Albermann K: Genome sequencing and analysis of the versatile cell factory Aspergillus niger CBS 513.88. Nature Biotech 2007, 25:221-231.

57. Galagan JE, Selker EU: RIP: the evolutionary cost of genome defense. Trends Genet 2004, 20:417-423.

58. Dürschner-Pelz V, Atkinson H: Recognition of Ditylenchus and other nematodes by spores of the endoparasitic fungus Verticillium balanoides. J Invertebr Pathol 1988, 51:97-106.

59. Felgenhauer BE: Techniques for preparing crustaceans for scanning electron microscopy. J Crustac Biol 1987, 7:71-76.

60. Li R, Yu C, Li Y, Lam TW, Yiu SM, Kristiansen K, Wang J: SOAP2: an improved ultrafast tool for short read alignment. Bioinformatics 2009, 25:1966-1967.

61. Haas BJ, Salzberg SL, Zhu W, Pertea M, Allen JE, Orvis J, White O, Buell CR, Wortman JR: Automated eukaryotic gene structure annotation using EVidenceModeler and the Program to Assemble Spliced Alignments. Genome Biol 2008, 9:R7.

62. Zhang Z, Carriero N, Zheng D, Karro J, Harrison PM, Gerstein M: PseudoPipe: an automated pseudogene identification pipeline. Bioinformatics 2006, 22:1437-1439.

63. Dyrløv Bendtsen J, Nielsen H, von Heijne G, Brunak S: Improved prediction of signal peptides: SignalP 3.0. J Mol Biol 2004, 340:783-795.

64. Katoh K, Standley DM: MAFFT multiple sequence alignment software version 7: improvements in performance and usability. Mol Biol Evol 2013, 30:772-780

65. Stamatakis A: RAxML-VI-HPC: maximum likelihood-based phylogenetic analyses with thousands of taxa and mixed models. Bioinformatics 2006, 22:2688-2690

66. Sanderson MJ: Estimating absolute rates of molecular evolution and divergence times: a penalized likelihood approach. Mol Biol Evol 2002, 19:101-109.

67. Lücking R, Huhndorf S, Pfister DH, Plata ER, Lumbsch HT: Fungi evolved right on track. Mycologia 2009, 101:810-822

68. Li H, Coghlan A, Ruan J, Coin LJ, Hériché J-K, Osmotherly L, Li R, Liu T, Zhang Z, Bolund L: TreeFam: a curated database of phylogenetic trees of animal gene families. Nucleic Acids Res 2006, 34(suppl 1):D572-D580.

69. De Bie T, Cristianini N, Demuth JP, Hahn MW: CAFE: a computational tool for the study of gene family evolution. Bioinformatics 2006, 22:1269-1271.

70. Edgar RC, Myers EW: PILER: identification and classification of genomic repeats. Bioinformatics 2005, 21(suppl 1):i152-i158.

71. Jurka J, Kapitonov W, Pavlicek A, Klonowski P, Kohany O, Walichiewicz J: Repbase Update, a database of eukaryotic repetitive elements. Cytogenet Genome Res 2005, 110:462-467.

72. Benson G: Tandem repeats finder: a program to analyze DNA sequences. Nucleic Acids Res 1999, 27:573.

73. Brenner S: The genetics of Caenorhabditis elegans. Genetics 1974, 77:71-94. 
74. Simms D, Cizdziel PE, Chomczynski P: TRIzol: A new reagent for optimal single-step isolation of RNA. Focus 1993, 15:99-102.

75. Conesa A, Nueda MJ: maSigPro User's Guide; 2009. http://bioc.ism.ac.jp/2.11/ bioc/vignettes/maSigPro/inst/doc/maSigPro-tutorial.pdf.

76. Mortazavi A, Williams BA, McCue K, Schaeffer L, Wold B: Mapping and quantifying mammalian transcriptomes by RNA-Seq. Nat Methods 2008, 5:621-628.

doi:10.1186/1471-2164-15-114

Cite this article as: Liu et al: Drechslerella stenobrocha genome

illustrates the mechanism of constricting rings and the origin of nematode predation in fungi. BMC Genomics 2014 15:114.

\section{Submit your next manuscript to BioMed Central and take full advantage of:}

- Convenient online submission

- Thorough peer review

- No space constraints or color figure charges

- Immediate publication on acceptance

- Inclusion in PubMed, CAS, Scopus and Google Scholar

- Research which is freely available for redistribution 